\title{
Robust Image-Based IVUS Pullbacks Gating
}

\author{
Carlo Gatta ${ }^{1}$, Oriol Pujol ${ }^{1}$, Oriol Rodriguez Leor ${ }^{2}$, \\ Josepa Mauri Ferre ${ }^{2}$, and Petia Radeva ${ }^{1}$ \\ ${ }^{1}$ Computer Vision Center, Campus UAB, Edifici O, 08193, Bellaterra, \\ Barcelona, Spain \\ cgatta@cvc.uab.es \\ ${ }^{2}$ Unitat d'hemodinàmica cardíaca hospital universitari Germans Trias i Pujol \\ Badalona, Spain
}

\begin{abstract}
Intracoronary UltraSound (IVUS) imaging allows to obtain high resolution images of internal part of coronary arteries. This tool is unique in the possibility to explore internal vessel structures of the coronary wall, being a powerful tool for diagnosis 1 . Since the coronary vessel is moving due to the periodical contraction and expansion of heart muscles, the acquired images present different artifacts. One of the most severe problems is the longitudinal oscillation of the IVUS catheter inside the vessel. To alleviate this problem, ECG-gating has been proposed. The goal of gating is to have subsequent frames that represent the internal vessel section in "stable" position and avoid the repetition of frames; that is to generate an image sequence in which the artifacts due to the heart beat have been removed while, possible translation due to vessel tortuosity can still be present. This paper presents a simple and efficient model of catheter longitudinal movement together with a fast and robust image based gating algorithm. Experimental results on 9 sequences from 7 patients, plus a comparison with ECG gating are presented.
\end{abstract}

\section{Introduction}

IVUS images are acquired with a constant speed motorized pullback of the ultrasound catheter thus providing a tool for potential applications able to compute longitudinal vessel/plaque measurements. However, IVUS pullbacks suffer from different artifacts. First of all, (1) the position of the transducer is not fixed with respect to the vessel morphology in the plane orthogonal to vessel extension. This cause that subsequent frames in a motorized pullback can be misaligned. Moreover, (2) since the visual aspect of the vessel can change due to its elasticity and due to the heart motion, the IVUS sampling depends on the sampling instant with respect to the heart cycle. This causes that subsequent frames in the pullback can have different vessel size or relative position and/or rotation in the image plane. Finally, (3) the longitudinal movement of the transducer is affected by a continuous oscillatory movement due to the heart beats; this causes that some positions in the vessel are sampled multiple times, and the pullback presents an oscillation in the longitudinal direction that can be easily seen in the resulting pullback video. The uneven sampling of the vessel structure (caused by

D. Metaxas et al. (Eds.): MICCAI 2008, Part II, LNCS 5242, pp. 518 5252008.

(C) Springer-Verlag Berlin Heidelberg 2008 
(3)) can be reduced using a gating method. The gating is thought as a method to sample evenly spaced and stable frames. The stability refers to the fact that, after the gating, in subsequent frames the vessel should have a similar position and rotation. This theoretically permits to have a sampling that does not contain artifacts due to the heart cyclic movement. The gating can be performed on-line, but it requires a longer time of IVUS intervention for the physician and for the patient. Another possibility is to perform a motorized pullback while capturing the ECG signal and then perform an off-line ECG gating. This technique heavily reduces the duration of the clinical exam while giving sub-optimal frames, since the frame rate is fixed, and we can sample only approximately at desired instants. Recently, other disadvantages of off-line ECG-gating have been highlighted: (1) the optimal sampling instant in the heart cycle is difficult to select (usually, the end-diastolic point) and it has high inter and intrapatient variability; (2) the ECG signal is a global measurement of the heart electrical activity while the artifacts due to the heart movement can change locally depending on the actual position of the catheter. These two points have been partially addressed in [2]. A new promising direction in IVUS off-line gating is to perform an image-based analysis of the pullback data and to infer optimal sampling points without considering the ECG signal.

\section{Previous Works on Image-Based IVUS Gating}

In 3] authors propose an algorithm that extracts the information on cardiac cycle by analyzing image sequence variation of two properties computed on a region of interest (ROI). The properties are the Average Intensity of the ROI and the Absolute Intensity Difference between ROIs of subsequent frames. In [4] authors discuss results of their Intelligate method showing that the method performs actually as good as an ECG gating technique. In [5] and [2] authors propose an image-based gating algorithm. The basic idea is to introduce the use of a dissimilarity matrix $\Phi\left(F_{i}, F_{j}\right)$ that measures the dissimilarity between the frames $F_{i}$ and $F_{j}$ of the same IVUS pullback, where $i$ and $j$ represent temporal variables. Once defined a proper measure such that $\Phi\left(F_{i}, F_{j}\right) \geq 0$, they obtain a matrix that exhibits a repetitive pattern of local minima and ridges of local maxima. The pattern is repeated so that some diagonals represent the loci of local minima, thus representing a specific interval between any two frames that present minimal dissimilarity 1 . The first diagonal of local minima is displaced a number of columns; this displacement is a clue for detecting the average heart beat along the sequence. To enhance the sharpness of maximal ridges, they convolutes the matrix $\Phi$ with an X-shaped inverted Gaussian kernel obtaining a matrix $\hat{D}$. Now, local maxima on the above defined diagonal identifies couple of frames that have a high similarity and minimal inter-frame motion. Then, authors use two algorithms to select the best frames in the path on the diagonal that have the highest local maxima in $\hat{D}$. In [6] authors propose a method to

$\overline{1}$ The principal diagonal is obviously not considered since $\Phi\left(F_{i}, F_{i}\right)=0$. 
extract the cardiac phase from IVUS sequences based on the hypothesis that the oscillation of the vessel wall is visible in longitudinal cuts.

\section{Our Method for Image-Based Gating}

In this section we show how the image-to-image distance based on Normalized Cross-Correlation in 2 has been substituted by the distance between image descriptors with the goal of increasing robustness. Moreover, we show a method to detect gating instants that is more simple and robust than the one presented in [2].

\subsection{Basic Hypothesis and Model of Catheter Movement}

The method we propose is based on the hypothesis that the image content of the IVUS pullback changes mainly for two reasons: (1) the catheter is moving through the vessel thus sampling different structures, (2) the heart cycle induces a periodic movement to the vessel both in longitudinal direction as well as non-rigid transformation of the vessel image in the short axis. These two facts contribute to the image content and we can assume that the image modifications due to the catheter movement (1) are slower than the ones produced by the periodic vessel movement and deformation (2). We can also assume that the modifications introduced by the cyclic heart movement are, in general, periodic. Let $V(l, x, y)$ be a virtual stack of all images that represents the vessel at each longitudinal position $l$. We can model the image formation as:

$$
I(t, x, y)=G\left(V\left(l_{0}+s \cdot t+f(\phi(t)), x, y\right), \phi(t)\right)
$$

where $s$ represents the constant speed of the motorized pullback, $f(\phi(t))$ represents an unknown function that describes the longitudinal displacement as a function of the heart cycle phase. $G$ represents an unknown $2 \mathrm{D}$ operator that describes the vessel image deformation due to the heart cycle, depending on the phase $\phi(t)$. The quantity $l_{0}+s \cdot t+f(\phi(t))$ represents the position 2 of the sensor in the vessel at time $t$. Figure 1 (right) presents a schematic simplified description of this movement. This formulation implicitly assumes that, for two instants $t_{1}, t_{2}$ with the same phase $\phi\left(t_{1}\right)=\phi\left(t_{2}\right)$, the difference between two images $I\left(t_{1}\right)$ and $I\left(t_{2}\right)$ depends only on the vessel content since the only variable that changes in equation (1) is the longitudinal translation $s \cdot t$. While this model is oversimplified, it catches the main behavior of the catheter and vessel movements. For the goals of gating, the estimation of $f(\phi(t))$ is the optimal solution to the problem. In next subsections we will propose an algorithm to find minima and maxima of $f(\phi(t))$ since the complete estimation of $f(\phi(t))$ is a difficult problem.

\subsection{Detailed Description of the Method}

To estimate minima and maxima of $f(\phi(t))$ we first build a dissimilarity matrix as in [2] but using image descriptors instead of image-to-image direct comparison.

\footnotetext{
${ }^{2}$ The equation represents the catheter position as an initial value $l_{0}$ plus a constant speed $s$ motion plus an unknown quantity $f$ that depends on the phase $\phi(t)$.
} 
Then, we propose a simple, effective and efficient way to analyze the dissimilarity matrix to obtain $f(\phi(t))$ minima and maxima. Summarizing, the steps are: (1) create proper descriptor for each image; (2) build a dissimilarity matrix $D$ between frames of the sequence using a distance metric between descriptors; (3) analyze the matrix $D$ to find a good sequence of instants $\left\{t_{1}, t_{2} \ldots t_{N}\right\}$ to sample, i.e. instants in which the displacements $\left\{f\left(\phi\left(t_{1}\right)\right), f\left(\phi\left(t_{2}\right)\right) \ldots f\left(\phi\left(t_{N}\right)\right)\right\}$ represent all the $f$ local minima or maxima. As a consequence of step (3), theoretically the artifacts due to the catheter longitudinal oscillation $f(\phi(t))$ and the unknown deformation $G$ are completely removed. The following sections describe the method in details.

Image descriptor: Subsequent IVUS images in a pullback can be quite different even if the content is very similar. This is due to different facts, among them: the image is corrupted by multiplicative noise, the viewpoint (the catheter) can change thus resulting in a translation and rotation (from the catheter point of view), the elasticity of the tissue and the temporarily dilation of the vessel can result in a non-rigid transformation. For these reasons we need an image descriptor that is invariant to translation and rotation (on the center of catheter), thus reducing the influence of the catheter roto-translation. Moreover, we need a descriptor that is robust to noise. To tackle the first property, we transform the IVUS image from Cartesian to polar coordinates, $I^{(P)}(r, \theta)=$ $I^{(C)}\left(x_{k}+r \cos (\theta), y_{k}+r \sin (\theta)\right)$ where $\left(x_{k}, y_{k}\right)$ is the center of both the catheter and the Cartesian image. For notational simplicity, from now on we refer to the polar image as $I(x, y)$. After this transformation, rotation on the catheter axis results in translation and translation results in deformation. Since the catheter position in the image plane varies slowly, this transformation can be sufficient to alleviate the problem of the catheter movement in the image plane. To have a descriptor with the required properties we use local filters, such as the Gabor filters, but summarize their response over the whole image. Even if this seems contradictory, the Gabor filters allow to locally measure the size and orientation of the textures in the image and the integration over the whole image gets rid of the possible noise simply by averaging. In this way, small variations are almost uninfluential while important and similar changes (e.g. same changes in the texture) become significant. The descriptor is a vector of $M$ elements $\boldsymbol{v}(t)=\left\{v_{1}(t), v_{2}(t), \ldots, v_{M}(t)\right\}$ based on the following formula:

$$
v_{i}=\frac{1}{X Y} \sum_{x=1}^{X} \sum_{y=1}^{Y}\left|I(x, y) * g_{i}(x, y)\right|
$$

where $g_{i}$ are Gabor patches which continuous component is null. The parameters of all $M=16$ Gabor patches are defined as 4 banks of increasing spatial frequency ( $\sigma$ from 12.72 to 1.59 in pixels and $F$ from 0.04 to 0.35 in cycles/pixel) combined with 4 banks of different orientations in steps of $\pi / 4$ ( $\theta$ from 0 to $3 \pi / 4)$.

Dissimilarity matrix: The dissimilarity matrix is a square matrix in which both the rows and columns represent the time axis. The matrix is represented 

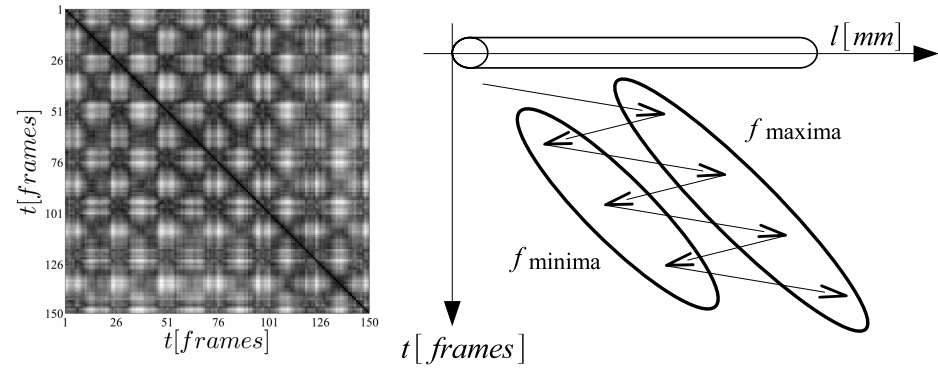

Fig. 1. Left: An example of matrix $D$. Darker values represent smaller values. Right: A schematic spatiotemporal representation of catheter movement.

by values defined as $D_{i, j} \equiv D(i, j)=d(\boldsymbol{v}(i), \boldsymbol{v}(j))$ where $i$ and $j$ are temporal variables and $d$ is a distance measure between descriptors. Different distance measures can produce different dissimilarity matrices. Among different choices, we used the Euclidean distance since it provides the sufficient sensitivity to descriptor variations and it is computationally feasible.

Catheter movement model and analysis of $D$ : Once built the dissimilarity matrix $D$, it is easy to notice that a regular repeating pattern is visible (see figure 1, left). This pattern is strictly related to the variations induced by the shifting function $f(\phi(t))$, the deformation operator $G$ and the translation $s \cdot t$ of the motorized pullback. While the effect of the deformation $G$ has been reduced by Cartesian to polar image transformation and by the use of summed Gabor responses, the effect of longitudinal translation $s \cdot t+f(\phi(t))$ is still present and influences the appearance of the matrix $D$. In matrix $D$, local minima indicated by, e.g. $\left(t_{1}, t_{2}\right)$ denote couples of similar frames $I\left(t_{1}, x, y\right) \equiv I_{1}$ and $I\left(t_{2}, x, y\right) \equiv I_{2}$. Theoretically, this means that the images $I_{1}$ and $I_{2}$ are two different sampling of two similar unknown images $V\left(l_{1}, x, y\right)$ and $V\left(l_{2}, x, y\right)$. Moreover, $I_{1}$ and $I_{2}$ can present unknown and different deformations $G$. To show an example of this fact, we selected a time $t_{1}$ in a pullback sequence and searched for local minima in the vector $D\left(t_{1}, j\right)$. Figure 2 shows $I_{1}$ and four images corresponding to the first four local minima in $D\left(t_{1}, j\right)$ starting from the diagonal. It can be noticed

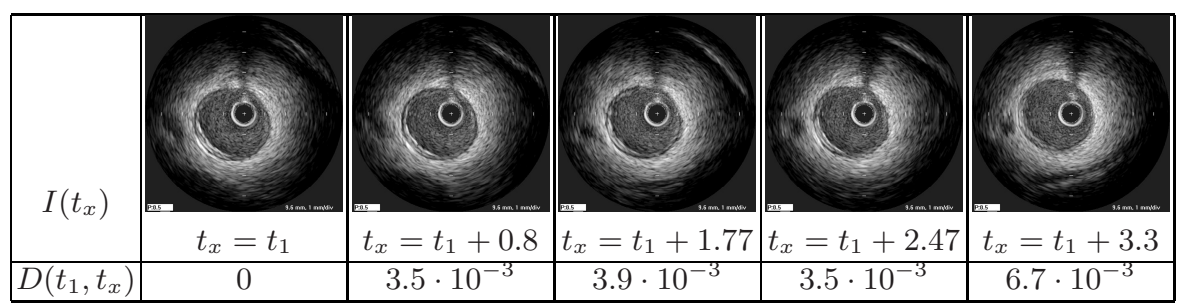

Fig. 2. Example of images with similar descriptors (times are in seconds) 
that the visual differences in images increase with the temporal delay, since the catheter is actually moving with an average constant speed $s$. In fact, the last image presents a dissimilarity value with respect to $t_{1}$ that is double the other images and shows effectively some novelties in the image structure (see e.g. the top right and bottom parts of the image). To obtain a robust estimation of the phase $\phi(t)$ and $f(\phi(t))$ is a difficult task thus we restrict our goal to find the points in which the function $f(\phi(t))$ has its maxima and minima. Figure 1 (right) shows a spatio-temporal graph in which the movement of the catheter is represented with respect to the vessel, showing the points of local $f(\phi(t))$ maxima and minima too. Identifying these two points is easier than estimating the whole function $f(\phi(t))$. Moreover, these two points indicate the instants in which the direction of the catheter motion is changing, and thus theoretically, where the real speed of the catheter is very small. For these reasons identifying these two points is good for gating IVUS pullbacks. Supposing that $t_{m}$ is an instant such that $f\left(\phi\left(t_{m}\right)\right)$ is a local maximum 3 , we can notice in figure 1 (right) that if we move forward on the time axis, we move backward on the $l$ axis (space) and if we move backward in time, we still move backward in $l$. This means that there are couples of sampled images of the vessel are almost similar in both sequences but in inverted order. This fact appears in the matrix D as lines of local minima parallel to the anti diagonal (see figure 1 for an example). Then, to detect these points we sum values along diagonals parallel to the anti diagonal, using proper normalization, obtaining a function $m(t)$ where local minima represent local maxima or minima of $f(\phi(t))$ :

$$
m(t)=\frac{1}{n} \sum_{\tau=1}^{n} D(t-1+\tau, t-\tau)
$$

where $n$ is the number of neighbor frames considered for the analysis. Usually, a value of $n>200$ is sufficient for having a good $m(t)$ signal. Figure 3 shows the function $m(t)$ computed on the matrix $D$ of figure 1 As it can be noticed, the local minima are clearly visible, thus it is easy to select optimal frames by tracking local minima. Next section provides a comparison with standard ECG gating in producing longitudinal cuts of several pullback sequences.

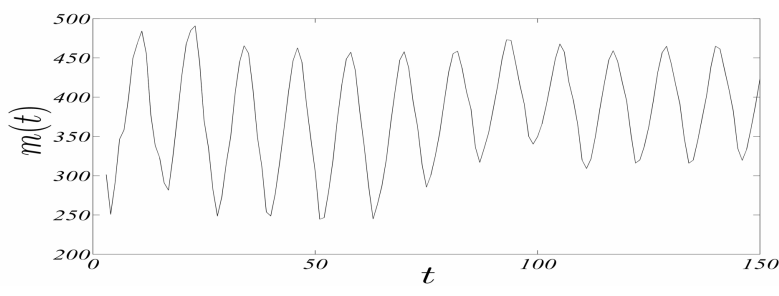

Fig. 3. Detection of $f$ minima and maxima (see text)

$\overline{3}$ This applies in a similar way to local minima. 


\section{Results}

We tested the proposed method on 9 sequences (approx. 2000 frames for each sequence) from 7 patients. Since a well defined methodology for comparison of gating algorithms is not available, and no rigorous validation of previous image based gating algorithm is accessible, we performed a comparison with ECG-gating. Figure 4 shows a comparison between an ECG-gated longitudinal cut (frames have been sampled at the end-diastolic point) and the proposed image-based gating algorithm. It can be noticed that the image-based gated longitudinal cut appears "smoother" than the ECG gated one. To quantitatively compare the proposed method to ECG gating we compare the number of selected frames and propose the following measure of horizontal discontinuity $S$ of a longitudinal cut $L(x, y)$ defined as the root mean square of the horizontal discrete derivative: $S(L)=\sqrt{\frac{1}{(X-1) Y} \sum_{x=1}^{X-1} \sum_{y=1}^{Y}(L(x+1, y)-L(x, y))^{2}}$. Table 1 shows the number of selected frames and values of the discontinuity measure $S$ for all the 9 sequences and for both ECG and image-based gated longitudinal cuts. The proposed method selects approximately $11 \%$ more frames than ECGgating due to the differences in phase between image based and ECG selected frames. This results in shorter detected loops for the proposed algorithm. While the discontinuity $S$ does not provide absolute quality measurement, it can be used to compare two different methods that produce images containing similar visible structures. In this case, the proposed method works similar or better than ECG gating for all tested sequences.

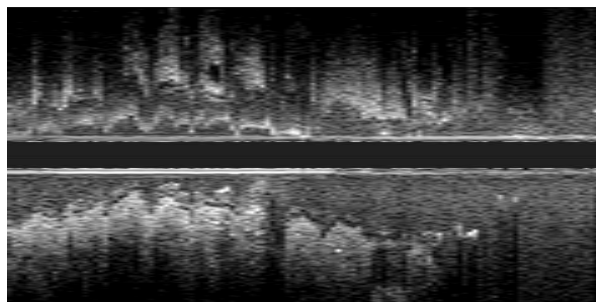

ECG gated

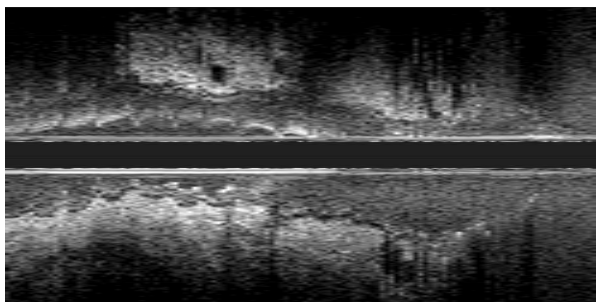

Image-based gated

Fig. 4. An example of ECG gated and image-based gated longitudinal cuts

Table 1. Number of selected frames and discontinuity measurement on 9 sequences for ECG and Image based gated longitudinal cuts

\begin{tabular}{|r|c|c|c|c|c|c|c|c|c||c|}
\hline Seq. n. & 1 & 2 & 3 & 4 & 5 & 6 & 7 & 8 & 9 & Mean \\
\hline ECG \# frames & 67 & 117 & 41 & 66 & 42 & 100 & 133 & 40 & 60 & 74 \\
Image-based \# frames & 74 & 130 & 53 & 75 & 57 & 111 & 144 & 40 & 57 & 82.33 \\
\hline ECG S & 0.131 & 0.122 & 0.140 & 0.140 & 0.135 & 0.129 & 0.114 & 0.145 & 0.148 & 0.133 \\
Image-based S & 0.125 & 0.106 & 0.129 & 0.130 & 0.117 & 0.124 & 0.111 & 0.146 & 0.137 & 0.125 \\
\hline
\end{tabular}




\section{Conclusion and Future Work}

In this paper we presented a simple and efficient model of catheter longitudinal movement together with a fast and robust image based gating algorithm. The model is necessary to understand the nature of catheter movement and the artifacts during the pullback acquisition procedure. Then, an efficient and robust algorithm is proposed. The use of image descriptors makes the algorithm robust and modular, since other descriptors can be incorporated into the algorithm. The method is not affected by the vessel deformation and ultrasound image noise, moreover, it has been tested on different IVUS sequences providing good results. As future directions, a deeper investigation on highly non-regular heart beats is also one of our next goals. The proposed algorithm can potentially be used as a pre-processing step to IVUS sequence for longitudinal measurements of vessel's structures.

\section{Acknowledgments}

This research is/was supported in part by the projects TIN2006-15308-C02, FIS PI061290.

\section{References}

1. Burke, A.P., Farb, A., hui Liang, G.T.M.Y., Smialek, J., Virmani, R.: Coronary risk factors and plaque morphology in men with coronary disease who died suddenly. The New England Journal of Medicine 336(18), 1276-1281 (1997)

2. O'Malley, S., Carlier, S., Naghavi, M., Kakadiaris, I.: Image-based frame gating of ivus pullbacks: A surrogate for ecg. IEEE International Conference on Acoustics, Speech and Signal Processing 1, 433-436 (2007)

3. Zhu, H., Oakeson, K.D., Friedman, M.H.: Retrieval of cardiac phase from IVUS sequences. In: Medical Imaging 2003: Ultrasonic Imaging and Signal Processing, vol. 5035 , pp. 135-146 (2003)

4. de Winter, S., Hamers, R., Degertekin, M., Tanabe, K., Lemos, P.A., Serruys, P.W., Roelandt, J.R., Bruining, N.: Retrospective image-based gating of intracoronary ultrasound images for improved quantitative analysis: The intelligate method. Catheterization and Cardiovascular Diagnosis 61(1), 84-94 (2004)

5. O'Malley, S., Naghavi, M., Kakadiaris, I.: Image-based frame gating for contrastenhanced ivus sequences. In: Proceedings of Computer Vision for Intravascular and Intracardiac Imaging (CVII), Copenhagen, Denmark (October 2006)

6. Barajas, J., Caballero, K.L., Rodriguez, O., Radeva, P.: Cardiac phase extraction in IVUS sequences using 1-D gabor filters. In: 29th Annual International Conference of the IEEE Engineering in Medicine and Biology Society 2007, pp. 343-346. IEEE, Los Alamitos (2007) 